\title{
Teaching and assessment of core residency competencies by Ophthalmic Clinical Evaluation Exercise (OCEX)
}

\author{
Rekha Khandelwal $^{1, *}$, Bijlani Madhavi², Raje Dhanajay ${ }^{3}$ \\ ${ }^{2}$ Junior Resident, ${ }^{3}$ Statistician, ${ }^{1}$ Professor \& HOD, ${ }^{1,2}$ Dept. of Ophthalmology, NKP Salve Institute of Medical Sciences \& Lata \\ Mangeshkar Hospital, Nagpur, Maharashtra, ${ }^{3}$ MDS Bioanalytics, Nagpur, India
}

*Corresponding Author:

Email: rekha.khandelwal@gmail.com

\begin{abstract}
Introduction: The Ophthalmic Clinical Evaluation Exercise (OCEX) checklist is like mini-CEX developed for ophthalmology by American Board of Internal Medicine's Clinical Evaluation Exercise. It is a validated checklist used to observe residentpatient interactions.

Most of the assessments during residency program are based on cognitive domain and lack psychomotor and affective domain. The present study assessed the utility of OCEX as a learning and assessment tool for ophthalmology residents.

Material and Methods: This study was carried out in ophthalmology department of NKP SIMS \& LMH Nagpur for a period of six months. It was a Quasi Experimental (Before and after) study. Seven ophthalmology residents (second or third year of residency) participated in the study. Each resident had three OCEX encounters, four weeks apart over the period of three months. Two faculty members were assessors for OCEX encounters. Data was collected on interview skills, clinical examination, interpersonal skills (professionalism) and case presentation. The performance was rated and feedback was shared with resident after every encounter.

Results: On analysing the results, it was found that there was a significant difference in the performance of residents between initial and final OCEX encounter. Statistical significance was measured using Friedman ANOVA test for difference in mean performance score between initial and final encounter. Faculty and students had good satisfaction with OCEX system of learning cum assessment tool.

Conclusion: The present study demonstrated that OCEX leads to clinical competency improvement in ophthalmology residents by providing critical formative feedback immediately after the encounter. The resident feedback towards OCEX was favourable.
\end{abstract}

Keywords: OCEX, Ophthalmology, Residents, Assessment tool, Competency.

\section{Introduction}

The residency years are the most important years for any specialty training where a post graduate student learns desired competencies in history taking, clinical examination, interpersonal skills, communication skills, and presentation of cases in that particular specialty. As most of the current assessments are based on cognitive domain and do not assess psychomotor and affective domain, post graduate students lack competencies in these areas.

The Ophthalmic Clinical Evaluation Exercise $(\text { OCEX })^{1,2}$ is a validated and reliable method used for quality improvement in ophthalmology residents. It facilitates supervised learning environment (SLE) involving direct observation of a doctor-patient clinical encounter by a junior resident to improve competencies in interview skills, clinical skills and professionalism through immediate and formative feedback.

It has shown to be content valid and reliable assessment strategy for improving clinical case presentation. The results are fairly reliable on three or four encounters. An advantage of OCEX is that it fulfils all the three basic requirements of assessment techniques which facilitates learning. 1) the content of the training program, the competencies expected as outcomes 2) trainee feedback after the encounter 3) the objective method of assessment steer trainee towards the desired outcomes. ${ }^{3}$

Competency based medical education(CBME) also target towards the programs which promotes adequate learner supervision. Traditionally, senior learners teach and supervise junior learners with increasing responsibility during training. Frequently, this occurs with limited faculty supervision. This activity is seen as critical to the learner's professional development and is believed to be a vital component of the learning community and culture of training programs (American Board of Internal Medicine 2009). ${ }^{4}$

It is important to understand that supervision for all levels of learners can enrich learning and will ensure the delivery of safe and effective patient care. Assessment of a student's competency for psychomotor and affective domain in ophthalmology is a real challenge for teachers. Eye being a sensory organ these two domains play a vital role in the management of patient who is visually compromised. Assessment should also balance issues of validity and reliability.

Many of the assessment methods are variations on the traditional oral examination which is time consuming and where no feedback is given to the students. In 1998, the Accreditation Council of Graduate Medical Education (ACGME) began an initiative, called the Outcome Project, which fostered 
residency training with a focus on development and assessment of the six competencies, including medical knowledge, patient care, interpersonal and communication skills, systems- based practice, professionalism, and practice-based learning and improvement. $^{5}$

Among the assessment tools targeted on various competencies evolving for years, the direct observation at workplace has played an important role in the process of these educational reforms. ${ }^{6}$

As proven by many studies that providing feedback to the students is most influential factor for their learning and achievement ${ }^{7}$. More attention should be paid on the assessment to improve learning.

Out of many assessment tools OCEX could be one of the tool which can be used for learning and assessment for the postgraduates in Ophthalmology. An OCEX assessment involves the observation of a patient-doctor encounter performed by the trainee. The key features of OCEX include assessment of interview skills, clinical examination, interpersonal skills and case presentation on actual patient and immediate feedback on his/her performance. The assessor's evaluation is recorded on a checklist which enables the assessor to provide verbal developmental feedback to the trainee. The data and feedback enable the learner to assess themselves and learn better. OCEX is generally led by the trainee i.e trainee chooses the patient, timing and assessor. In USA, the assessment of residents, and increasingly of students as well, is largely based on a model that was developed by the Accreditation Council for Graduate Medical Education (ACGME) ${ }^{8}$.This model uses six areas of competence and some means of assessing them (ACGME Outcome Project 2000).These are medical knowledge, patient care (including clinical reasoning), professionalism, interpersonal and communication skills, practice-based learning and improvement (including information management) and systems-based practice (including health economics and teamwork).

Hence, the present study was planned to facilitate competence in six vital areas of learning and assessing them using OCEX.

\section{Aim}

To study effectiveness of Ophthalmic Clinical Evaluation Exercise (OCEX) as a teaching cum assessment tool for core competencies in Ophthalmology residents

\section{Material and Methods}

Ethical considerations: An approval was sought from the institutional review board (IRB) before starting the study. Informed consent was taken from the residents before inclusion in the study.

Study design: Quasi experimental (before \& after) study
Study duration and area: Department of Ophthalmology of NKP Salve Institute of Medical Sciences, Nagpur for a period of 6 months from March 2016 to September 2016.

Study population: Residents of department of Ophthalmology

Inclusion criteria and exclusion criteria: All residents who gave consent were included in the study.

Sample size: The current batch strength of residents undergoing training under Ophthalmology departments at NKP Salve Institute of Medical Sciences, Nagpur in the academic session 2016-17 is 11 . Since, we targeted residents who had finished minimum twelve months of training in Ophthalmology to be able to train on OCEX, we included all junior residents of second and third year (Seven). After explaining to them the purpose of the study a written informed consent was taken. All seven residents (by convenience sampling method) and two assessors (faculty) consented to participate and were enrolled in the study.

Sampling and randomization: Out of the seven junior residents (JR2 and JR3) who consented to be included in the study three were then randomly assigned to assessor 1 and the other four were assigned assessor 2 .

Study tool: OCEX form (Annexure 1)

The pre-validated Ophthalmic Clinical Evaluation Exercise (OCEX) checklist was used for second and third year residents in Ophthalmology. OCEX is developed by International Council Ophthalmology (ICO) to assess a resident's patient care skills, medical knowledge, and interpersonal skills.

Step 1: After sensitizing the assessor and residents to the OCEX tool, after 2 weeks, the principal investigator and an evaluator observed the resident-patient interaction for $15-20 \mathrm{~min}$ and gave performance feedback on OCEX using following scale:
1. Does not meet expectations
2. Meets some expectations
3. Meets all expectations
4. Exceed expectations na: not applicable

Step 2: The assessor used OCEX encounters for three such sessions, four weeks apart, over period a period of three months. The feedback was given after every encounter.

The perception of the residents and faculty on OCEX as a method of teaching and learning was obtained after the last encounter.

\section{Statistical Analysis}

The ratings on different questions related to interview, examination, professional and presentation skills were obtained by students and summarized in terms of mean, median and inter-quartile range (IQR) for each stage. The comparison of ratings and total score across stages was performed for each question and the statistical significance was obtained using 
Friedman ANOVA. Significance was evaluated at 5\% level and the analysis was carried out using SPSS ver 20.0 software.

Flow chart

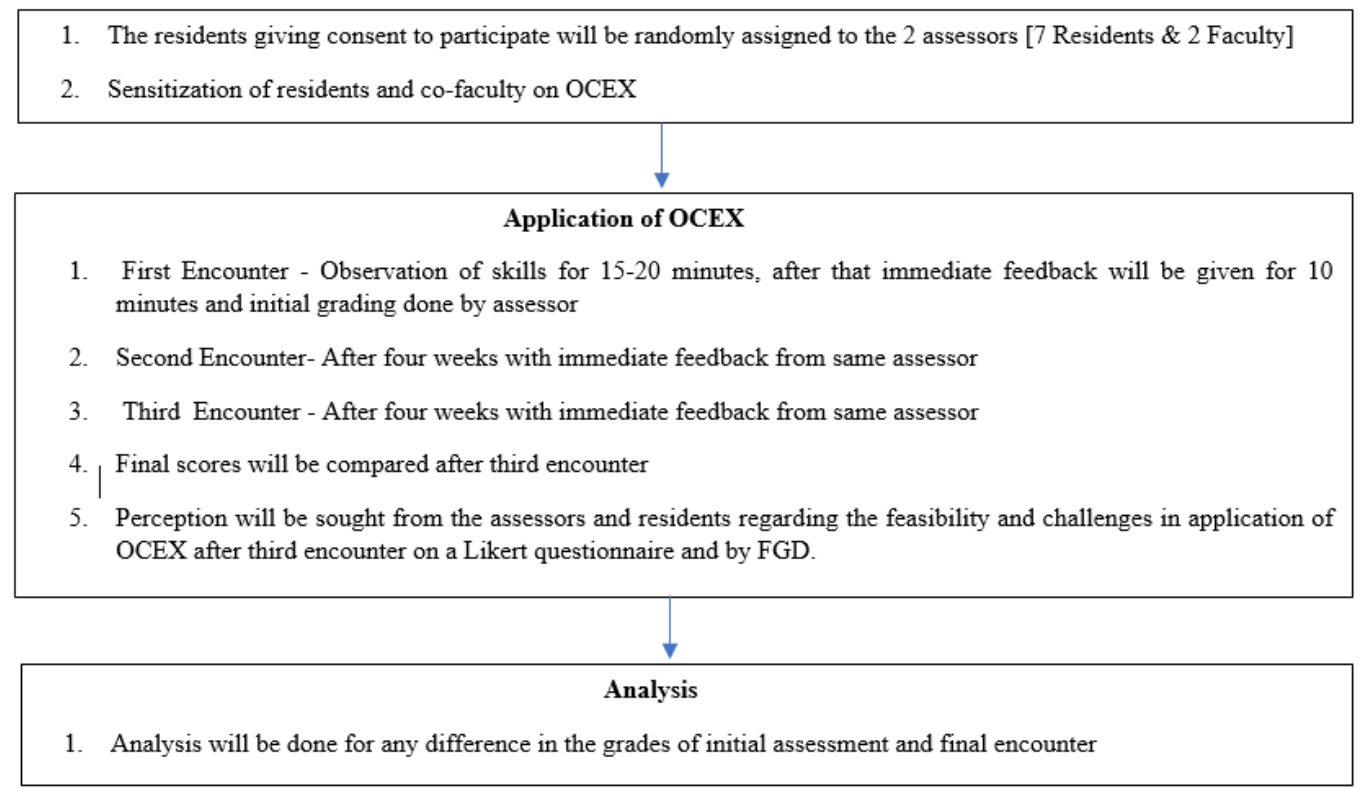

\section{Results}

Seven Ophthalmology residents participated in the study of which 2 were males and 5 were females. All the residents were assessed for various skills related to Ophthalmology i.e. interview skills, examination, interpersonal skills and case presentation. The skills were assessed using the OCEX tool.

Table 1 provides the descriptive statistics like mean, median and inter-quartile range (IQR) for ratings obtained on different questions related to interview skills at three distinct time points. A comparison of score distribution across times was performed for each question, which resulted into statistical significance ( $\mathrm{p}$ $<0.05$ ) using Friedman ANOVA. The median score for each question increased at follow up 1 as compared to baseline (initial), and then further increased or remained same at follow up 2, as compared follow up 1. The median total score at follow up 2 (47) was significantly higher than that of follow up 1 (41) and baseline (28). Fig. 1a depicts the change of median scores across time points for each question through line plots, while Fig. $1 \mathrm{~b}$ gives the column chart of mean scores for each question at baseline and final follow up.

Table 1: Descriptive statistics for ratings obtained on different questions related to interview skills

\begin{tabular}{|l|c|c|c|c|}
\hline \multirow{2}{*}{$\begin{array}{c}\text { Interview } \\
\text { Skills }\end{array}$} & \multicolumn{3}{|c|}{ Mean [Median (IQR)] } & \multirow{2}{*}{ P-value* } \\
\cline { 2 - 4 } Q1 & Initial & Follow Up 1 & Follow Up 2 & \\
\hline Q2 & $2.86[3(0.5)]$ & $3.86[4(0)]$ & $3.86[4(0)]$ & 0.008 \\
\hline Q3 & $2.43[2(1)]$ & $3.57[4(1)]$ & $4.00[4(0)]$ & 0.002 \\
\hline Q4 & $2.00[2(1)]$ & $3.14[3(0)]$ & $4.00[4(0)]$ & 0.002 \\
\hline Q5 & $2.43[2(1)]$ & $3.43[3(1)]$ & $4.00[4(0)]$ & 0.002 \\
\hline Q6 & $1.86[2(0)]$ & $3.00[3(0)]$ & $4.00[4(0)]$ & 0.003 \\
\hline Q7 & $2.00[2(0)]$ & $3.29[3(0.5)]$ & $3.86[4(0)]$ & 0.001 \\
\hline Q8 & $2.00[2(1)]$ & $3.43[3(1)]$ & $3.71[4(0.5)]$ & 0.001 \\
\hline Q9 & $2.57[3(1)]$ & $3.43[3(1)]$ & $4.00[4(0)]$ & 0.002 \\
\hline Q10 & $2.29[2(1)]$ & $2.71[3(0.5)]$ & $3.71[4(0.5)]$ & 0.004 \\
\hline Q11 & $2.43[2(1)]$ & $2.86[3(0.5)]$ & $4.00[4(0)]$ & 0.004 \\
\hline Q12 & $2.86[3(0)]$ & $4.00[4(0)]$ & $4.00[4(0)]$ & 0.004 \\
\hline Total score & $28.43[28(1.5)]$ & $40.14[41(3.5)]$ & $47.14[47(1.5)]$ & 0.001 \\
\hline
\end{tabular}

*Obtained using Friedman ANOVA; P-values in bold indicate statistical significance 


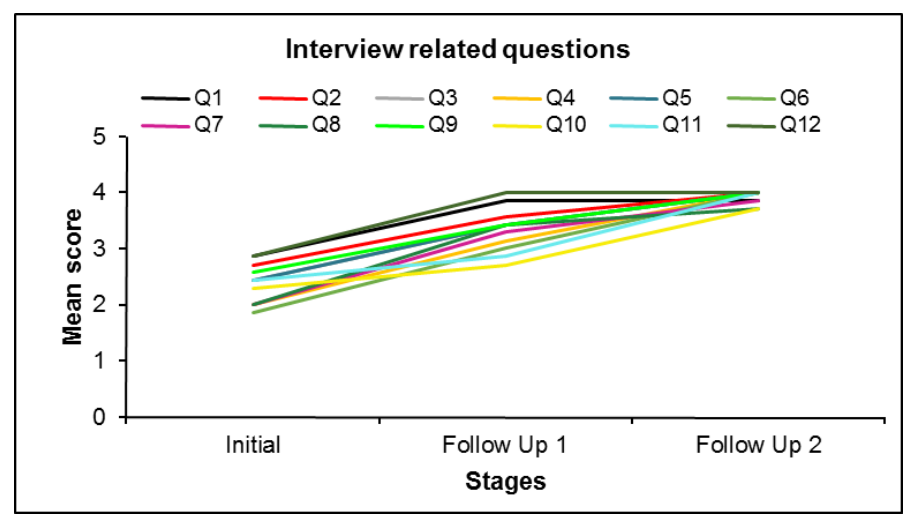

Fig. 1a: Line plot showing change of mean scores on questions related to interview skills across stages

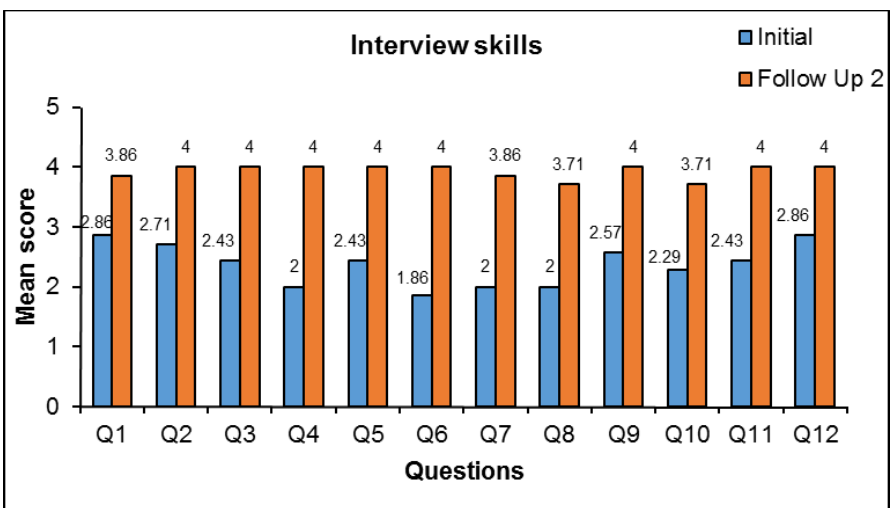

Fig. 1b: Column chart showing mean scores on questions related to interview skills at initial stage and final follow up 2

On similar lines, Table 2 provides the mean, median and IQR values for questions related to examination. It is evident that the median scores across time points for each question were statistically significantly different $(\mathrm{p}<$ 0.05). The median scores increase with time and were maximum at final follow up 2 for each question. The overall score at final follow up (31) was significantly higher than follow up 1 (25) and baseline (17). Fig. 2a provides the line chart for examination related questions across time, while Fig. $2 \mathrm{~b}$ gives the column chart of mean scores at baseline and follow up 2.

Table 2: Descriptive statistics for ratings obtained on different questions related to Examination

\begin{tabular}{|l|c|c|c|c|}
\hline \multirow{2}{*}{ Examination } & \multicolumn{3}{|c|}{ Mean [Median (IQR)] } & \multirow{2}{*}{ P-value* } \\
\cline { 2 - 4 } & Initial & Follow Up 1 & Follow Up 2 & \\
\hline Q1 & $2.71[3(0.5)]$ & $4.00[4(0)]$ & $4.00[4(0)]$ & 0.001 \\
\hline Q2 & $2.29[2(0.5)]$ & $3.43[3(1)]$ & $4.00[4(0)]$ & 0.001 \\
\hline Q3 & $1.71[2(0.5)]$ & $3.00[3(0)]$ & $4.00[4(0)]$ & 0.001 \\
\hline Q4 & $2.43[2(1)]$ & $3.00[3(0)]$ & $4.00[4(0)]$ & 0.001 \\
\hline Q5 & $2.57[3(1)]$ & $3.71[4(0.5)]$ & $4.00[4(0)]$ & 0.002 \\
\hline Q6 & $2.00[2(0)]$ & $3.14[3(0)]$ & $4.00[4(0)]$ & 0.001 \\
\hline Q7 & $1.57[2(1)]$ & $2.57[3(1)]$ & $3.43[3(1)]$ & 0.002 \\
\hline Q8 & $1.57[2(1)]$ & $2.43[2(1)]$ & $3.57[4(1)]$ & 0.002 \\
\hline Total score & $16.86[17(2)]$ & $25.29[25(2)]$ & $31.00[31(0)]$ & 0.001 \\
\hline
\end{tabular}

*Obtained using Friedman ANOVA; P-values in bold indicate statistical significance 


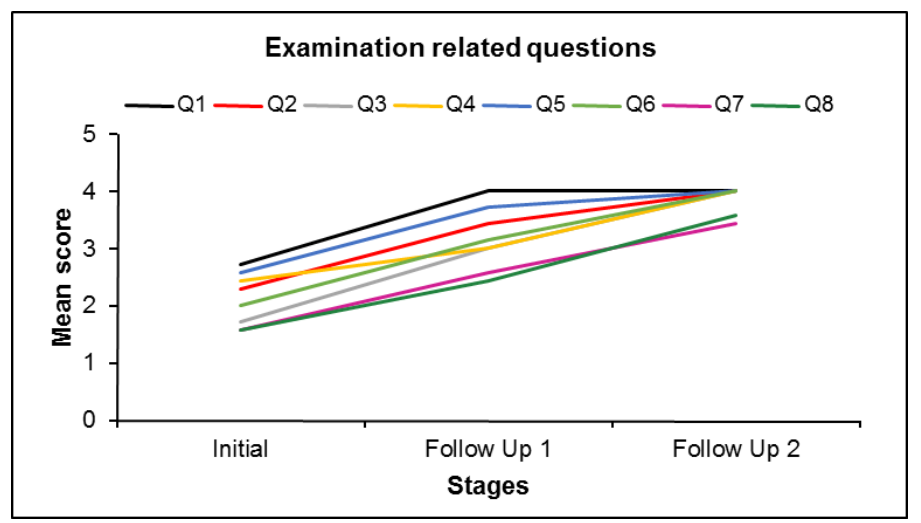

Fig. 2a: Line plot showing change of mean scores on questions related to examination across stages

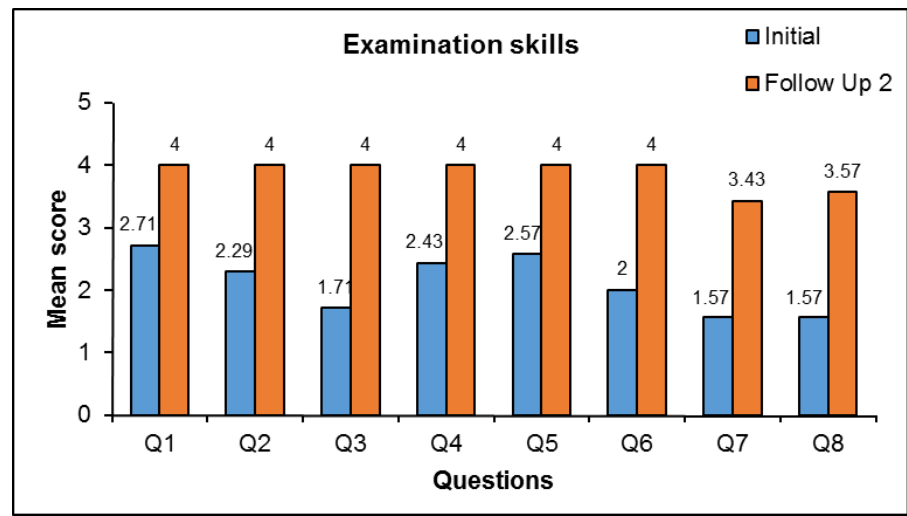

Fig. 2b: Column chart showing mean scores on questions related to examination skill at initial and final follow up 2

Table 3 provides the descriptive statistics for questions related to professional skills. It is evident that the median scores across time points for each question were statistically significantly different $(\mathrm{p}<0.05)$. The median scores either increased with time or were constant in the follow up. The overall score at final follow up (27) was significantly higher than follow up 1 (22) and baseline (15). Fig. 3a provides the line chart for median scores of questions related to professional skills across time, while Fig. 3b gives the column chart of mean scores at baseline and follow up 2.

Table 3: Descriptive statistics for ratings obtained on different questions related to Professional skills

\begin{tabular}{|l|c|c|c|c|}
\hline \multirow{2}{*}{$\begin{array}{c}\text { Professional } \\
\text { skills }\end{array}$} & \multicolumn{3}{|c|}{ Mean [Median (IQR)] } & \multirow{2}{*}{ P-value* } \\
\cline { 2 - 5 } Q1 & $2.57[3(1)]$ & $3.43[3(1)]$ & $4.00[4(0)]$ & 0.003 \\
\hline Q2 & $2.57[2(1)]$ & $3.86[4(0)]$ & $3.71[4(0.5)]$ & 0.010 \\
\hline Q3 & $2.86[3(0.5)]$ & $3.57[4(1)]$ & $4.00[4(0)]$ & 0.008 \\
\hline Q4 & $1.86[2(0.5)]$ & $3.00[3(0)]$ & $4.00[4(0)]$ & 0.002 \\
\hline Q5 & $1.86[2(0)]$ & $2.86[3(0)]$ & $4.00[4(0)]$ & 0.001 \\
\hline Q6 & $1.71[2(0.5)]$ & $2.86[3(0)]$ & $3.71[4(0.5)]$ & $\mathbf{0 . 0 0 1}$ \\
\hline Q7 & $1.71[2(1)]$ & $3.29[3(1)]$ & $3.71[4(0.5)]$ & $\mathbf{0 . 0 0 4}$ \\
\hline & & $\begin{array}{l}22.86 \\
(2.5)]\end{array}$ & $27.14[27(0)]$ & $\mathbf{0 . 0 0 1}$ \\
\hline
\end{tabular}

*Obtained using Friedman ANOVA; P-values in bold indicate statistical significance 


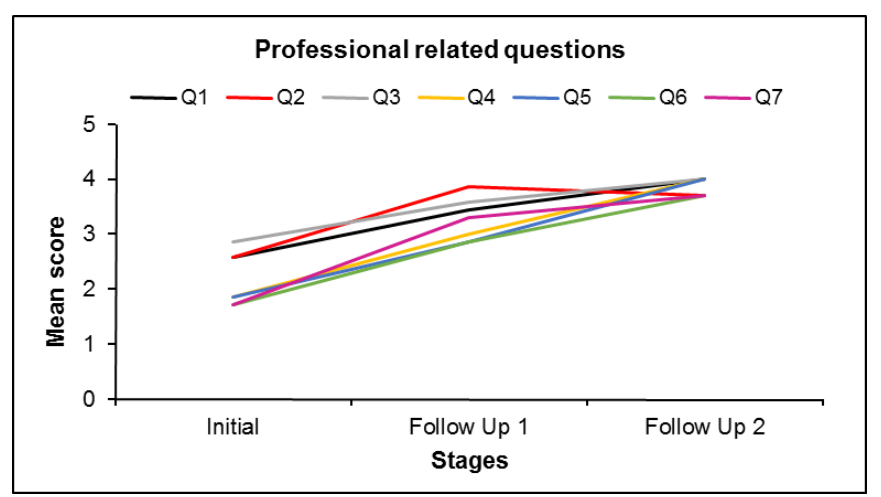

Fig. 3a: Line plot showing change of mean scores on questions related to professional skills across stages

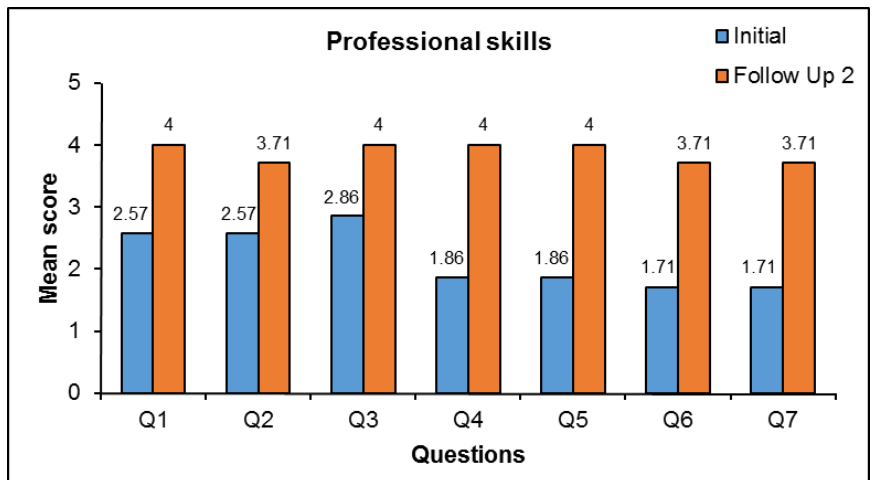

Fig. 3b: Column chart showing mean scores on questions related to professional skills at initial and final follow up 2

Table 4 provides the descriptive statistics for questions related to case presentation. It is evident that the median scores across time points for each question were statistically significantly different $(p<0.05)$. The median scores either increased with time or were constant in the follow up. The overall score at final follow up (23) was significantly higher than follow up 1 (20) and baseline (13). Fig. 4a provides the line chart for median scores of questions related to case presentation across time, while Fig. $4 \mathrm{~b}$ gives the column chart of mean scores at baseline and follow up 2.

Table 4: Descriptive statistics for ratings obtained on different questions related to Case presentation

\begin{tabular}{|c|c|c|c|c|}
\hline \multirow{2}{*}{$\begin{array}{c}\text { Case } \\
\text { Presentation }\end{array}$} & \multicolumn{3}{|c|}{ Mean [Median (IQR)] } & \multirow{2}{*}{ P-value* } \\
\hline & Initial & Follow Up 1 & Follow Up 2 & \\
\hline Q1 & $2.43[2(1)]$ & $4.00[4(0)]$ & $4.00[4(0)]$ & 0.001 \\
\hline $\mathrm{Q} 2$ & $2.14[2(0)]$ & $3.00[3(0)]$ & $4.00[4(0)]$ & 0.001 \\
\hline$\overline{\mathrm{Q} 3}$ & $2.00[2(0)]$ & $2.86[3(0)]$ & $4.00[4(0)]$ & 0.001 \\
\hline $\mathrm{Q} 4$ & $2.29[2(0.5)]$ & $3.43[3(1)]$ & $4.00[4(0)]$ & 0.003 \\
\hline $\mathrm{Q} 5$ & $1.86[2(0)]$ & $3.00[3(0)]$ & $3.43[3(1)]$ & 0.002 \\
\hline Q6 & $2.57[3(1)]$ & $3.71[4(0.5)]$ & $4.00[4(0)]$ & 0.001 \\
\hline Total score & $13.29[13(1.5)]$ & $20.00[20(0.5)]$ & $23.43[23(1)]$ & 0.001 \\
\hline
\end{tabular}

*Obtained using Friedman ANOVA; P-values in bold indicate statistical significance

Table 5: Descriptive statistics for overall ratings obtained on different questions

\begin{tabular}{|l|c|c|c|l|}
\hline \multirow{2}{*}{ Overall skills } & \multicolumn{3}{|c|}{ Mean [Median (IQR)] } & \multirow{2}{*}{ P-value* } \\
\cline { 2 - 5 } & Initial & Follow Up 1 & Follow Up 2 & \\
\hline Grand Total & $73.71[73(3.5)]$ & $108.29[110(5.5)]$ & $128.71[129(1)]$ & 0.001 \\
\hline
\end{tabular}

*Obtained using Friedman ANOVA; P-values in bold indicate statistical significance 


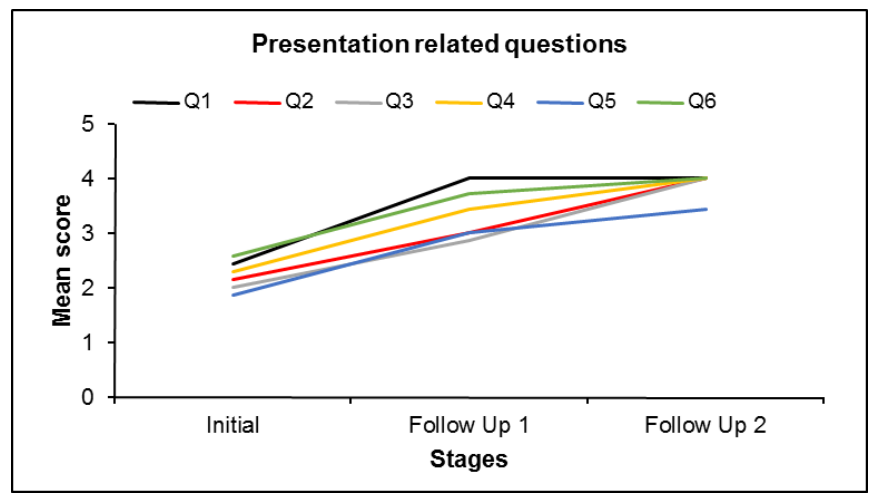

Fig. 4a: Line plot showing change of mean scores on questions related to Case Presentation across stages

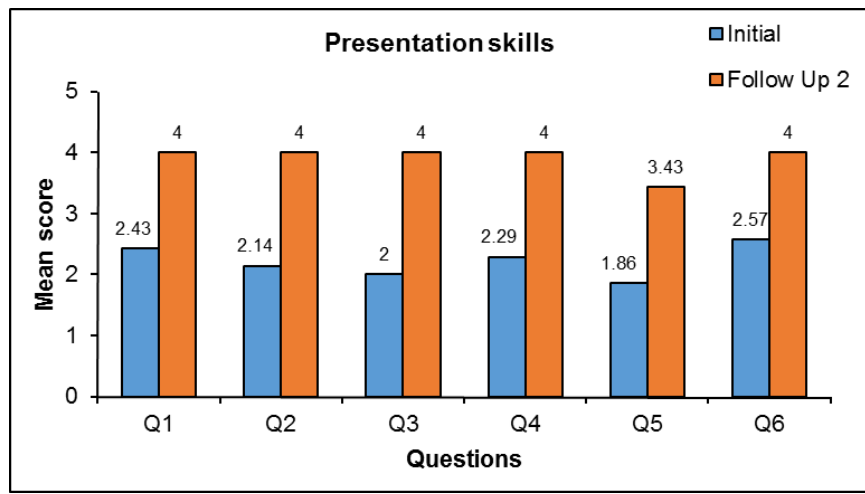

Fig. 4b: Column chart showing mean scores on questions related to Case Presentation skill at initial and final follow up 2

The grand total was obtained at all the three encounters and compared statistically. The difference of score distribution was significant across times with $\mathrm{p}$ value of 0.001 . The median at follow up 2 (129) was significantly higher than that of follow up 1 (110) and baseline (73).

\section{Discussion}

Medical education currently is changing from the traditional teaching to competency-based medical education (CBME). ${ }^{9}$

The old style of medical education was originally outlined by Abraham Flexner in 1910, to a competencybased system of education and it is referred to as "Structure/Process" education. ${ }^{10}$

There are four components - (1) identifying the outcomes; (2) defining performance levels for each competency; (3) developing a framework for assessing competencies; and (4) continuous evaluation of the CBME program to see if it is indeed producing the desired outcomes.

Recent advances in medical technology have identified mini-CEX, as a tool for learning and assessing students. Evaluation refers to the interpretation of data as it relate to the utility of a curriculum. Although Mini-CEX is likely to play an increasingly important role in competency assessment over time as the direct observation of learners providing care will remain a cornerstone of assessment and evaluation process.

As Carraccio and colleagues $(2002)^{9}$ have noted, competency-based education requires greater involvement by the faculty because of the need for direct observation, feedback and increased frequency and quality of formative assessment. 


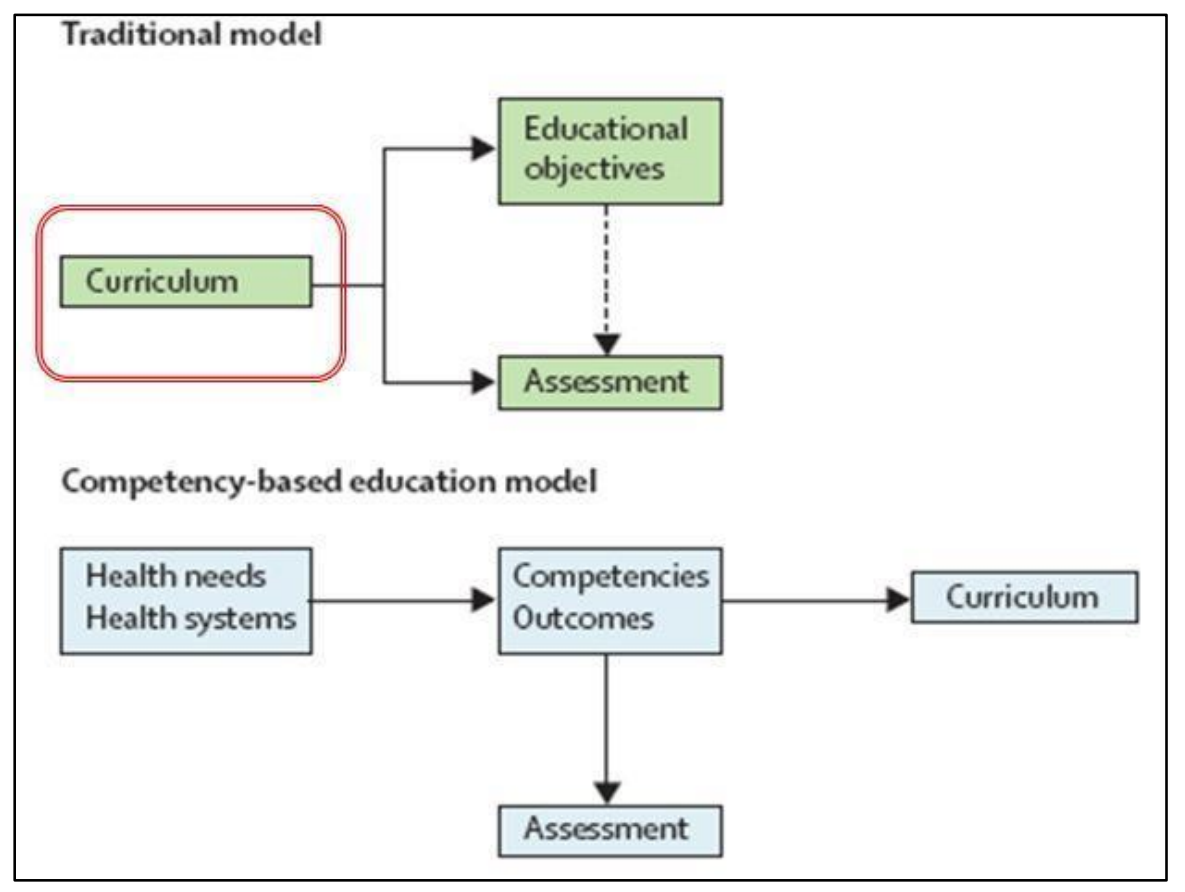

Competence builds on a foundation of basic clinical skills, scientific knowledge and moral development. Epstein and Hundert proposed a definition of professional competence as the habitual and judicious use of communication, knowledge, technical skills, clinical reasoning, emotions, values and reflection in daily practice for the benefit of the individual and community being served. ${ }^{11}$

The American Board of Internal Medicine distinguished between the four different dimensions of clinical competence, where problem solving was the core aspect. ${ }^{12}$ These included abilities (i.e. knowledge, technical skills and interpersonal skills), problemsolving skills (i.e. data gathering and diagnosis), the nature of the medical illness (problems encountered by physicians) and social and psychological aspects of the patients' problems. In a later report, more elements were added-communication skills, professionalism (e.g. ethical practice, understanding diversity, responsible attitude), and system-based practice (i.e. understanding of the healthcare system to improve and optimize healthcare). ${ }^{13}$

Assessment of a student's actual performance after graduation and post-graduation in the consulting rooms is a real challenge for teachers. Increasing attention is being placed on this type of assessment (highest level of Miller's pyramid) because of its possible high consequential and predictive Validity. Attempts at performance assessment have to balance issues of validity and reliability.

OCEX: The CEX (clinical evaluation exercise) was developed as a way to assess internal medicine residents in America. It required direct observation of a resident obtaining a complete history and performing examination. The problems encountered with CEX in internal medicine included long hours needed for it, limited reliability and inter-rater variability. ${ }^{14}$ Due to this The American Board of Internal Medicine devised the mini-CEX which is a shorter version of CEX .It is also used to provide immediate formative feedback to the resident. The mini-CEX has been used as a learning tool and evaluation device for medical students. ${ }^{15,16}$

The American board has assigned valid and reliable tools to assess resident competence in 6 general areas: (1) medical knowledge, (2) patient care, (3) practice-based learning, (4) interpersonal and communication skills, (5) professionalism, and (6) systems-based practice. ${ }^{8}$ Surgery is considered as a seventh competency for ophthalmology programs by the American Board of Ophthalmology. ${ }^{1}$

An OSCE uses direct observation of a resident performing an examination on a standardized patient. But the time required is a major drawback to this method. Most of the Ophthalmology programs have relatively small resident group and hence OSCE seems to be expensive method in terms of time and money. ${ }^{1}$

The OCEX attempts to combine the comprehensiveness of CEX and shorter duration and feedback of mini-CEX in one assessment tool. It assesses ACGME patient care requirements, professionalism, interpersonal skills and medical knowledge competency. Thus, it has been validated and suggested by panel of experts to be implemented for ophthalmology residents, as it is a brief method for assessing four vital out of seven general competencies. It also allows to provide a critical, instant and formative feedback to the resident. ${ }^{1}$

The ACGME has suggested that assessment tools should have an alpha greater than 0.8. The OCEX alpha was found to be 0.81 . The alpha statistics for the 
interpersonal skills/professionalism and case presentation subscales were 0.73 and 0.70 , respectively. Although these do not reach the alpha value of 0.80 , it is agreed that a value of 0.70 for a newly developed scale is still acceptable. ${ }^{2}$

The alpha statistic provides us with an indication of how well the items in a scale hang together as one unit and reliably measure the underlying construct of interest. But looking at the OCEX, one could argue that a student may be quite competent at asking about the history of a patient's present illness, but not be good about asking the patient about pain. Similarly, a student may be proficient in slit-lamp examination, but not on a patient's motility assessment. In both situations, an assessor would rate the student high in one area and low in another, resulting in a low alpha statistic. Therefore, the low alpha statistics for these scales might not be indicating a poor performance per se, but rather it may suggest that in addition to using the OCEX to assess a resident's competence in carrying out these skills, additional objective methods should also be used. Or, if time is limited for conducting the resident evaluation, perhaps the evaluator should use the OCEX to assess only the interpersonal skills/professionalism and case presentation skills and assess the resident's interview and examination skills using a more objective manner.

We used OCEX in the present study to sensitize the students and staff to this method of learning cum assessment tool. We also compared the results of initial encounter with final encounter for each area.

In the present study we found that for interview skills, the median score for each question increased at first follow up encounter were better than baseline (initial) encounter, and they further increased or remained same at second follow up encounter. The median total score at follow up 2 (47) was significantly $(\mathrm{p}<0.05)$ higher than that of follow up 1 (41) and baseline (28).

Similarly, the median scores for clinical examination skills increased with time and were maximum at final encounter for each question. The overall score at final follow up (31) was significantly higher $(\mathrm{p}<0.05)$ than follow up $1(25)$ and baseline (17). As there are very few studies on OCEX which are reported in literature we could not compare our results point by point with earlier studies. We compared with mini-CEX studies reported earlier. A previous study using mini-CEX also reported a statistically significant improvement in cognitive knowledge on comparing the pre-test and post-test results $(67.35 \pm 15.25$ versus $81.22 \pm 10.34, p<0.001)$ after application of mini CEX. ${ }^{17}$

Related to interpersonal skills and professionalism in our study we noted that the overall mean score at final follow up (27) was significantly higher than follow up 1 (22) and baseline (15). The median scores of questions related to professional skills across time also improved significantly in our study.
We also studied clinical case presentation and judgement. It was evident that the median scores for each question were statistically significantly significant $(p<0.05)$. The median scores either increased with time or were constant in the follow up. The overall score at final follow up (23) was significantly higher than follow up 1 (20) and baseline (13).

In the present study, a total score was also obtained for all four assessment parameters and mean performance scores at all three encounters were compared. The difference of score distribution was significant across times with p-value of 0.001 . The median at follow up 2 (129) was significantly higher than that of follow up 1 (110) and baseline (73).

A study reported in the literature by Kogan JR et al on implementation of mini-CEX to evaluate medical students' interview skills, clinical skills, professionalism and clinical judgement improved significantly after 3 months of mini-CEX encounters. ${ }^{16}$

In the present study none of the resident /assessors rated mini CEX as unsatisfactory. Similarly, mini-CEX used for common primary eye care disorders in internship program also found that interns and faculty were satisfied with this type of assessment. ${ }^{18}$

The OCEX does require an assessor to observe patient resident encounter which may take 30-45 minutes. Many faculty members had concern over this. Hence to decrease the time requirement, residents might be evaluated on only one out of four subscales of OCEX at one time (i.e. interview skills, clinical examination, professionalism and case presentation). Future research will be able to highlight advantages and disadvantages of OCEX in a more elaborate manner.

As OCEX targets "does" level of Miller's pyramid, it can be applied as an appropriate method of resident assessment tool enhancing their competency.

\section{Conclusion}

The OCEX is the first assessment tool available with good validity and reliability to assess four of the seven ACGME competencies for ophthalmology. It also provides critical formative feedback after the encounter. Most residents and faculty members expressed their willingness to implement the OCEX for enhancing the competency-based skills in ophthalmology.

\section{Recommendation}

Medical educational unit should incorporate the OCEX (mini-CEX) as teaching-learning tool to promote competency based medical education in all departments.

\section{Acknowledgments}

We are grateful to the assessors and Junior Residents (2017-18) who volunteered to be part of this study. 
We would also express sincere thanks to the in-charge Medical Education Unit of NKP Salve Institute of Medical Sciences and Research Centre Nagpur.

\section{References}

1. Golnik KC,Goldenhar LM, Gittinger JW Jr,Lustbader JM: The Ophthalmic Clinical Evaluation Exercise (OCEX). Ophthalmol 2004;111(7):1271-74.

2. Golnik, K.C. and Goldenhar, L: The Ophthalmic Clinical Evaluation Exercise. Reliability determination. Ophthalmol 2005;112:1649-54.

3. John Norcini, Vanessa Burch: Workplace-based assessment as an educational tool: AMEE Guide No. 31 . Med Teacher 2007;29:855-71.

4. William F. Iobst, Jonathan Sherbino, Olle Ten Cate, Denyse L. Richardson, Deepak Dath, Susan R. Swing et al: Competency-based medical education in postgraduate medical education: Med Teacher 2010;32:651-56.

5. Swing SR: The ACGME outcome project: retrospective and prospective. Med Teacher 2007;29(7):648-54.

6. Pelgrim EA, Kramer AW, Mokkink HG, van den Elsen L, Grol RP, van der Vleuten CP: In training assessment using direct observation of single patient encounters: a literature review. Adv Health Sci Educ Theory Pract 2011;16(1):131-42.

7. Veloski J, Boex JR, Grasberger MJ, Evans A, Wolfson DB: Systematic review of the literature on assessment, feedback and physicians' clinical performance: BEME Guide No. 7. Med Teacher 2006;28:117-28.

8. ACGME and ABMS. Toolbox of assessment methods. A product of the joint initiative of the ACGME Outcome Project of the Accreditation Council for Graduate Medical Education (ACGME), and the American Board of Medical Specialties (ABMS). Version 1.1, September 2000
9. Carraccio C, Wolfsthal SD, Englander R, Ferentz K, Martin C: Shifting paradigms: From Flexner to competencies. Acad Med 2002;77:361-67.

10. 10.Frank JR, Snell LS, Ten Cate O, et al. Competencybased medical education: Theory to practice. Med Teacher 2010;32:638-45.

11. Epstein RM, Hundert EM. Defining and assessing professional competence. JAMA 2002;287:226-35.

12. American Board of Internal Medicine. Clinical competence in internal medicine. Ann Intern Med 1979;90:402-11.

13. American Board of Internal Medicine (ABIM). Residents: Evaluating your clinical competence: New competencies for Internal Medicine. Philadelphia, Pennsylvania: American Board of Internal Medicine Clinical Competence Program, Sept 2001-June 2002.

14. Holmboe ES, Hawkins RE. Methods for evaluating the clinical competence of residents in internal medicine: a review. Ann Intern Med 1998;129:42-8.

15. Hauer KE. Enhancing feedback to students using the Mini- CEX (Clinical Evaluation Exercise). Acad Med 2000;75:524.

16. Kogan JR, Bellini LM, Shea JA. Implementation of the Mini- CEX to evaluate medical students' clinical skills. Acad Med 2002;77:1156 -57.

17. Kuo-Chen Liao, Shou-Jin Pu, Maw-Sen Liu, Chih-Wei Yang, Han-Pin Kuo. Development and implementation of a mini-Clinical Evaluation Exercise (mini-CEX) program to assess the clinical competencies of internal medicine residents: from faculty development to curriculum evaluation. BMC Medical Education 2013, 13:31.

18. Kapoor Harpreet. Competency based structured internship program for enhanced clinical skills in managing common primary eye care disorders Available at http://www.faimer.org/education/fellows/abstracts/08Kap oor.pdf Assessed on 01/08/2016

\section{Annexure 1: Ophthalmic Clinical Evaluation Exercise (OCEX)}

The OCEX is an observed encounter between a resident and a new patient. The evaluator should be present in the exam room for the entire interaction. The intent is to rate the resident in all the categories listed below compared to a graduating resident and then provide immediate performance feedback. The rating system is:

1 - Does Not Meet Expectations 3 - Meets All Expectations

2 - Meets Some Expectations 4 - Exceeds Expectations na - Not Applicable

\begin{tabular}{|c|c|c|c|c|c|c|c|c|c|c|c|}
\hline & \multicolumn{6}{|c|}{ Interview Skills } & & & & & \\
\hline 1. Introduced self & 1 & 2 & 3 & 4 & na & 7. Review of systems & 1 & 2 & 3 & 4 & na \\
\hline 2. Obtained chief complaint & 1 & 2 & 3 & 4 & na & \begin{tabular}{|l|} 
8. Med list \\
\end{tabular} & 1 & 2 & 3 & 4 & na \\
\hline 3. History of present illness & 1 & 2 & 3 & 4 & na & \begin{tabular}{|l} 
9. Past medical history \\
. Pal
\end{tabular} & 1 & 2 & 3 & 4 & na \\
\hline 4. Pertinent negatives & 1 & 2 & 3 & 4 & na & 10. Social history & 1 & 2 & 3 & 4 & na \\
\hline 5. Pain inquiry & 1 & 2 & 3 & 4 & na & \begin{tabular}{|l|}
11. Family history \\
\end{tabular} & 1 & 2 & 3 & 4 & $\mathrm{Na}$ \\
\hline \multirow[t]{2}{*}{ 6. Allergies } & 1 & 2 & 3 & 4 & na & 12. Washed hands & 1 & 2 & 3 & 4 & $\mathrm{Na}$ \\
\hline & \multicolumn{6}{|c|}{ Examination } & & & & & \\
\hline 1. Best corrected $\mathrm{Va}$ & 1 & 2 & 3 & 4 & na & 5. External & 1 & 2 & 3 & 4 & na \\
\hline 2. Pupils / RAPD & 1 & 2 & 3 & 4 & na & 6. SLE & 1 & 2 & 3 & 4 & na \\
\hline 3. Visual Fields & 1 & 2 & 3 & 4 & na & $\begin{array}{|lr|}7 . & \text { IOP } \\
\text { gonioscopy) }\end{array}$ & 1 & 2 & 3 & 4 & na \\
\hline \multirow[t]{2}{*}{ 4. Motility } & 1 & 2 & 3 & 4 & na & 8. Funduscopy & 1 & 2 & 3 & 4 & na \\
\hline & \multicolumn{6}{|c|}{ Interpersonal Skills / Professionalism } & & & & & \\
\hline
\end{tabular}




\begin{tabular}{|c|c|c|c|c|c|c|c|c|c|c|c|}
\hline 1. Empathetic & 1 & 2 & 3 & 4 & na & 5. Explained diagnosis & 1 & 2 & 3 & 4 & na \\
\hline 2. Respectful \& courteous & 1 & 2 & 3 & 4 & na & $\begin{array}{l}6 . \\
\text { plan/options }\end{array}$ & 1 & 2 & 3 & 4 & na \\
\hline $\begin{array}{l}\text { 3. Used language the pt } \\
\text { Understands }\end{array}$ & 1 & 2 & 3 & 4 & na & $\begin{array}{l}\text { 7. Asked if patient had } \\
\text { questions }\end{array}$ & 1 & 2 & 3 & 4 & na \\
\hline 4. Explained findings & 1 & 2 & 3 & 4 & na & & & & & & \\
\hline & \multicolumn{6}{|c|}{ Case Presentation } & & & & & \\
\hline 1. Concise \& clear & 1 & 2 & 3 & 4 & na & $\begin{array}{l}\text { 4. Appropriate } \\
\text { differential Dx }\end{array}$ & 1 & 2 & 3 & 4 & $\overline{\text { na }}$ \\
\hline 2. Pertinent facts & 1 & 2 & 3 & 4 & na & 5. Appropriate plan & 1 & 2 & 3 & 4 & na \\
\hline 3. Pertinent pos \& negs & 1 & 2 & 3 & 4 & na & $\begin{array}{l}6 . \quad \text { Response to } \\
\text { attending's questions/ } \\
\text { suggestions }\end{array}$ & 1 & 2 & 3 & 4 & $\overline{\text { na }}$ \\
\hline
\end{tabular}

\section{Comments:}

We have reviewed this OCEX together. Resident initials:

Evaluator initials:

Date:

\begin{tabular}{|c|c|c|c|c|}
\hline \multicolumn{5}{|c|}{ Interpersonal Skills } \\
\hline & $\begin{array}{c}1 \\
\text { Does not meet }\end{array}$ & $\begin{array}{c}2 \\
\text { Meets some expectations }\end{array}$ & $\begin{array}{c}3 \\
\text { Meets all expectations }\end{array}$ & $\begin{array}{c}4 \\
\text { Exceeds all expectations }\end{array}$ \\
\hline Respectful & Disrespectful & $\begin{array}{l}\text { Curt, does not listen to all of } \\
\text { patient's questions/concerns }\end{array}$ & $\begin{array}{l}\text { Listens to patient, responds to patient } \\
\text { questions/concerns }\end{array}$ & $\begin{array}{l}\text { Extremely attentive to patient's questions, } \\
\text { concerns }\end{array}$ \\
\hline Understandable & $\begin{array}{l}\text { Constantly uses } \\
\text { medical jargon the } \\
\text { patient doesn't } \\
\text { understand }\end{array}$ & $\begin{array}{l}\text { Occasionally uses medical jargon } \\
\text { the patient doesn't understand }\end{array}$ & $\begin{array}{l}\text { Avoids or explains medical terms when } \\
\text { used }\end{array}$ & $\begin{array}{l}\text { Avoids or explains medical terms when used } \\
\text { and frequently asks whether they are } \\
\text { understood }\end{array}$ \\
\hline $\begin{array}{l}\text { Explained } \\
\text { Findings }\end{array}$ & No explanation & Cursory explanation & Thoroughly explained all pertinent findings & $\begin{array}{l}\text { Thoroughly explained al findings and used } \\
\text { models/photos }\end{array}$ \\
\hline $\begin{array}{l}\text { Explained } \\
\text { Diagnosis }\end{array}$ & No explanation & Cursory explanation & Thoroughly explained diagnosis & $\begin{array}{l}\text { Thoroughly explained diagnosis and used } \\
\text { models/photos }\end{array}$ \\
\hline Explained Plan & No explanation & Cursory explanation & Thoroughly explained plan & $\begin{array}{l}\text { Thoroughly explained plan and scheduled } \\
\text { tests }\end{array}$ \\
\hline $\begin{array}{l}\text { Asked if Patient } \\
\text { Had Questions. }\end{array}$ & Does not ask & $\begin{array}{l}\text { Asked if patient had questions but } \\
\text { didn't answer completely }\end{array}$ & $\begin{array}{l}\text { Asked if patient had questions and } \\
\text { answered questions thoroughly }\end{array}$ & $\begin{array}{l}\text { Asked if patient \& family had questions. And } \\
\text { answered thoroughly. Gave phone \# for } \\
\text { patient to call with questions }\end{array}$ \\
\hline \multicolumn{5}{|l|}{ Case Presentation } \\
\hline Concise/Clarity & Unintelligible & Somewhat Disorganized & Clear, concise, organized & Meticulous, exact, succinct but complete \\
\hline Pertinent Facts & Omits pertinent facts & Omits minor supporting facts & Covers all pertinent facts & $\begin{array}{l}\text { Covers all pertinent facts and omits all } \\
\text { irrelevant data }\end{array}$ \\
\hline $\begin{array}{l}\text { Pertinent } \\
\text { Positives \& } \\
\text { Negatives }\end{array}$ & Does not mention & $\begin{array}{l}\text { Mentions some pertinent positives } \\
\text { \& negatives }\end{array}$ & Covers all pertinent positives \& negatives & $\begin{array}{l}\text { Covers all pertinent positives \& negatives, } \\
\text { and omits irrelevant data }\end{array}$ \\
\hline $\begin{array}{l}\text { Differential } \\
\text { Diagnosis }\end{array}$ & Does not mention & $\begin{array}{l}\text { Provides basic but incomplete } \\
\text { differential } D x\end{array}$ & Provides appropriate differential Dx & Exhaustive differential $D x$ and cites literature \\
\hline Appropriate Plan & Does not mention & Provides basic but incomplete plan & Provides appropriate plan & Provides detailed plan and cites literature \\
\hline $\begin{array}{l}\text { Response to } \\
\text { Attending }\end{array}$ & Inappropriate & Listens but little response & Listens and responds appropriately & $\begin{array}{l}\text { Responds appropriately and cites relevant } \\
\text { literature }\end{array}$ \\
\hline
\end{tabular}




\begin{tabular}{|c|c|c|c|c|}
\hline \multicolumn{5}{|c|}{ OCEX Scoring Rubric } \\
\hline & $\begin{array}{c}1 \\
\text { Does notmeet }\end{array}$ & $\begin{array}{c}2 \\
2 \\
\text { Meets some expectations }\end{array}$ & $\begin{array}{c}3 \\
\text { Meets all expectations }\end{array}$ & $\begin{array}{c}4 \\
\text { Exceeds Expectations }\end{array}$ \\
\hline \multicolumn{5}{|l|}{ Interview Skills } \\
\hline Introduction & $\begin{array}{l}\text { Does notintroduce } \\
\text { him/her seff }\end{array}$ & $\begin{array}{l}\text { Introduces self as Dr. not as } \\
\text { resident }\end{array}$ & Introduces self as resident physician & $\begin{array}{l}\text { Introduces self to patient \& family and shakes } \\
\text { hands }\end{array}$ \\
\hline Chief Complaint & Does not elicita CC & $\begin{array}{l}\text { Elicits CC butlacks relevant } \\
\text { details. }\end{array}$ & Elicits CC \& details & Elicits CC and subtle, relevant details \\
\hline HPI & Does not elicit HPI & HPI lacks relevant details & HPl indudes most important details & HPl includes all relevant details \\
\hline $\begin{array}{l}\text { Pertinent } \\
\text { Negatives }\end{array}$ & $\begin{array}{l}\text { Does not elicit pertinent } \\
\text { negatives }\end{array}$ & Elicits some pertinent negatives & Elicts important pertinent negatives & Elicits even subtle pertinent negatives \\
\hline Pain Inquiry & Does not elicit. & Pain is elictied, not characterized & Elicits scaled rating of pain (0-10) & $\begin{array}{l}\text { Elicits scaled rating/ relieving/exacerbating } \\
\text { factors }\end{array}$ \\
\hline Allergies & Does not elicit. & $\begin{array}{l}\text { Elicits medical allergies without } \\
\text { symptom detail }\end{array}$ & $\begin{array}{l}\text { Elicits medical allergies with symptom } \\
\text { detall }\end{array}$ & $\begin{array}{l}\text { Elicits medical \& environmental } \\
\text { allergies/yymptoms }\end{array}$ \\
\hline ROS & Does not elicit. & Elicits incomplete ROS & Elicts most important items in ROS & Leaves no stone unturned \\
\hline Medication List & Does not elicit. & Obtains list, no dosages lfrequency & Obtains list with dosages ifrequency & Obtains list of meds \& herbal remedies \\
\hline Social History & Does not elicit. & Omits important details & Obtains important details & Elicts even subtle relevant details \\
\hline Family History & Does not elicit. & Omits important details & Obtains important details & Obtains subtle relevant details of family tree \\
\hline Hand Washing & Does not wash hands. & Washes his/her hands, no soap & Washes hands with soap & Washes hands before and after encounter \\
\hline \multicolumn{5}{|l|}{ Exam } \\
\hline Visual Acuity & Does not check & Checks, but not best corrected & Checks best corrected & $\begin{array}{l}\text { Does additional, appropriate testing relevant } \\
\text { to patien't's historylexam }\end{array}$ \\
\hline Pupils & Does not check & $\begin{array}{l}\text { Checks light reaction, does not } \\
\text { swing light }\end{array}$ & Checks light reaction and for RAPD & $\begin{array}{l}\text { Does additional, appropriate testing relevant } \\
\text { to patient's historylexam }\end{array}$ \\
\hline Visual Field & Does not check & $\begin{array}{l}\text { Confrontational VF done but } \\
\text { incompletely }\end{array}$ & $\begin{array}{l}\text { Confrontational visual fields done } \\
\text { correctly }\end{array}$ & $\begin{array}{l}\text { Does additional, appropriate testing relevant } \\
\text { to patient's historylexam }\end{array}$ \\
\hline Motility & Does not check & Checks ductions or versions & $\begin{array}{l}\text { Checks ductions / versions and alignment } \\
\text { in primary position }\end{array}$ & $\begin{array}{l}\text { Does additional, appropriate testing relevant } \\
\text { to patient's historylexam }\end{array}$ \\
\hline External & Does not check & Observes without measurements & Checks lid fissures \& for proptosis & $\begin{array}{l}\text { Does additional, appropriate testing relevant } \\
\text { to patient's historylexam }\end{array}$ \\
\hline SLE & Does not check & $\begin{array}{l}\text { Doesn'tcheck all depths of AC } \\
\text { andlor checks only } 1 \text { eye }\end{array}$ & $\begin{array}{l}\text { Checks both eyes, entire anterior } \\
\text { segment }\end{array}$ & $\begin{array}{l}\text { Does additional, appropriate testing relevant } \\
\text { to patient's historylexam }\end{array}$ \\
\hline IOP & Does not check & Poor applanation technique & Checks IOP correctly OU & $\begin{array}{l}\text { Does additional, appropriate testing relevant } \\
\text { to patien't's historylexam }\end{array}$ \\
\hline Fundus & Does not check & Indirect or slit lamp biomicroscopy & Indirect and slit lamp biomicroscopy & $\begin{array}{l}\text { Does additional appropriate testing relevant } \\
\text { to patien't's historylexam }\end{array}$ \\
\hline
\end{tabular}

\section{Descriptors of competencies demonstrated during the OCEX}

\section{Medical Interviewing Skills:}

Facilitates patient's telling of story; effectively uses questions/ directions to obtain accurate, adequate information needed; responds appropriately to affect, non-verbal cues.

Humanistic Qualities/Professionalism: Shows respect, compassion, empathy, establishes trust; attends to patient's needs of comfort, modesty, confidentiality, information.

Clinical Judgment: Selectively orders/performs appropriate diagnostic studies, considers risks, benefits.

Counselling Skills: Explains rationale for test/treatment, obtains patient's consent, educates/counsels regarding management.

Overall Clinical Competence: Demonstrates judgment, synthesis, caring, effectiveness, efficiency

Annexure 2:

Patient informed consent form

Patient identification number for this trial: 
Title of project: Teaching and assessment of core residency competencies by Ophthalmic Clinical Evaluation Exercise (OCEX)

Name of Principal Investigator: Dr Rekha Khandelwal

Mobile No.09823261794

The contents of the information sheet "Teaching and assessment of core residency competencies by Ophthalmic Clinical Evaluation Exercise (OCEX)"

As a teaching learning tool in enhancing skills of residents dated $15^{\text {th }}$ May 2016 that was provided have been read carefully by me / explained in detail to me, in a language that I comprehend, and I have fully understood the contents. I confirm that I have had the opportunity to ask questions. The nature and purpose of the study and its potential risks / benefits and expected duration of the study and other relevant details of the study have been explained to me in detail. I understand that my participation is voluntary and that I am free to withdraw at any time, without giving any reason. I understand that the information collected about me from my participation in this research may be looked at by responsible individuals. I give permission for these individuals to have access to my records. I agree to take part in the above study.

Name of the participant

Address:

Mobile: Date:

Signature:

This is to certify that the above consent has been obtained in my presence.

Signature of Principal Investigator

Date:

Place: NKP SIMS, Nagpur

Signature Witness -1

Date:

Place: NKP SIMS, Nagpur

Signature Witness - 2

Date:

Place: NKP SIMS, Nagpur 